\title{
Emerging FMS-like tyrosine kinase 3 inhibitors for the treatment of acute myelogenous leukemia
}

\author{
Hillary Prescott, PharmD, BCOP, Hagop Kantarjian, MD, Jorge Cortes, MD, and Farhad \\ Ravandi, MD ${ }^{\dagger}$ \\ The University of Texas, M.D. Anderson Cancer Center, Department of Leukemia, Houston, TX, \\ USA
}

\begin{abstract}
Introduction-The FMS-like tyrosine kinase 3 (FLT3) is highly expressed in acute leukemias. Mutations involving FLT3 are among the most common molecular abnormalities in acute myelogenous leukemia (AML). Available evidence suggests that these molecular lesions confer a shorter disease-free survival and overall survival in patients with intermediate-risk cytogenetics. Therefore, substantial interest in FLT3 as a therapeutic target has led to the development of several promising inhibitors that target this tyrosine kinase.
\end{abstract}

\begin{abstract}
Areas covered-This review covers the molecular pathways associated with FLT3 activation in patients with AML, the biological rationale for inhibiting FLT3 and recent clinical progress with FLT3 inhibitors for the treatment of AML. Six FLT3 inhibitors undergoing clinical evaluation are discussed. A review of selected published manuscripts on the subject of FLT3 inhibition in AML and a search of the English language manuscripts in PubMed using the index words FLT3 and AML were conducted and articles of interest selected.
\end{abstract}

Expert opinion-Mutated forms of FLT3, specifically FLT3-internal tandem duplication, have a significant impact on the prognosis of AML patients, particularly those with a normal karyotype. Inhibiting FLT3 may lead to clinical benefit for patients with AML. Newly developed FLT3 inhibitors have shown encouraging activity as monotherapy and in combination with other therapeutic agents.

\section{Keywords}

acute myelogenous leukemia; FLT3; FLT3 inhibitor; FLT3-ITD Expert

\section{Background}

Acute myelogenous leukemia (AML) is a heterogeneous disease characterized by aberrant proliferation of myeloid precursor cells that have lost their ability to differentiate [1]. Several patient- and disease-related factors play a role in determining the prognosis of patients with AML. Until recently, cytogenetics provided the most accurate prognostic

(C) 2011 Informa UK, Ltd. All rights reserved

${ }^{\dagger}$ Author for correspondence, The University of Texas, M.D. Anderson Cancer Center, Department of Leukemia, 1515 Holcombe Blvd. Box 428, Houston, TX 77030, USA, Tel: +1 713745 0394; Fax: +1 713794 4297, fravandi@ mdanderson.org. 
information related to overall survival (OS). However, recently identified molecular abnormalities such as mutations of nucleophosmin 1 (NPMI), CCAAT/enhancer-binding protein a (CEBPA) and FMS-like tyrosine kinase 3 (FLT3) genes provide important clinical and prognostic information. More specifically, FLT3 is a member of the class III receptor tyrosine kinase (RTK) family that includes FMS, KIT, and platelet-derived growth factor receptors $\alpha$ and $\beta$. Structural similarities among class III RTKs include five immunoglobulin-like domains in the extracellular region and an intracellular tyrosine kinase domain (TKD) that is interrupted by a sequence of amino-acid residues [2]. FLT3 is expressed by normal bone marrow cells and early progenitor cells [3] and is involved with differentiation, proliferation and survival of hematopoietic stem cells, as well as lymphoid and dendritic progenitor cells and cells of the immune system [4,5]. FLT3 expression has also been detected in tissues other than early progenitor cells but to a much lesser degree [6].

The function of FLT3 can be defined by the activity of its ligand. Binding of FLT3 ligand (FL) to the receptor induces receptor dimerization which triggers receptor autophosphorylation [7], thereby, enabling the receptor to activate three major downstream pathways, signal transducer and activator of transcription 5 (STAT5), RAS/MAPK and PI3K/AKT. These pathways contribute to cell differentiation, proliferation and survival [8-12].

FLT3 is also highly expressed in AML, B-lineage acute lymphoblastic leukemia (ALL) and to a lesser extent in T-lineage ALL [3,13,14]. Activating mutations involving FLT3 are among the most common molecular abnormalities associated with AML and occur in $30 \%$ of adult patients with AML. The two most common activating mutations occur in the juxtamembrane domain (JM) and the TKD. Disruption of the JM by internal tandem duplications (ITD) is detected in $20-25 \%$ of AML cases and at a low frequency in myelodysplastic syndrome [15,16]. These mutations are always in frame and are 3 to 400 or more base pairs in length. Previously thought to be confined to the JM domain, a recent analysis of 753 FLT3-ITD-positive AML cases revealed the presence of this mutation type in the non-JM domain of 216 (28.7\%) patients [17]. FLT3-ITD mutations promote constitutive activation of FLT3 that leads to aberrant activation of the STAT5, RAS/MAPK and PI3K/AKT signaling pathways in the absence of the FL [18]. Additionally, $\sim 7-10 \%$ of adult AML cases have point mutations within the activation loop of the second kinase domain (FLT3-TKD). In a majority of cases, these mutations involve the substitution of tyrosine for aspartic acid at residue 835 (D835Y) [19-21]. Other activating point mutations have been identified in the TKD [22-24] and JM domain [25]. Similar to ITD mutations, point mutations lead to constitutive activation of FLT3 and aberrant activation of downstream signaling pathways, thereby, contributing to the pathogenesis of AML.

\section{Medical need}

AML is a heterogeneous disorder characterized by the proliferation of undifferentiated hematopoietic progenitor cells [1] and primarily afflicts the older population [26]. The prognosis of AML depends on the patient's age, performance status, karyotype, history of antecedent hematologic disease and initial response to treatment. Among these prognostic factors, the karyotype profile provides the most important prognostic information used to 
predict the response to induction therapy, relapse rate and OS $[27,28]$. However, the outcomes of patients with intermediate-risk cytogenetics are quite variable. The role of molecular abnormalities has given us some insight into this variability. For instance, NPM1 and $C E B P A$ mutations appear to confer a more favorable prognosis for patients with normal karyotype AML (NK-AML) [29], whereas mutations involving FLT3 in NK-AML are associated with shorter disease-free survival (DFS), remission duration and OS [30-32]. In cases where NPM1 and FLT3 are both mutated, the favorable outcomes normally conferred by mutated NPMI are negated [29]. Additionally, several published reports describe high frequencies of FLT3 mutations in acute promyelocytic leukemia (APL) and AML with a $\mathrm{t}(6 ; 9)(\mathrm{p} 23 ; \mathrm{q} 34)$ translocation [30-35]. Consistent with previous reports, a recent retrospective analysis of FLT3 mutations in newly diagnosed AML patients treated at The University of Texas M.D. Anderson Cancer Center revealed worse event-free survival (EFS) and OS in patients with NK-AML who harbored a FLT3-ITD mutation [36]. However, the presence of FLT3-ITD or FLT3-TKD in patients with favorable (core-binding factor) and poor-risk cytogenetics AML was not associated with worse EFS and OS. Furthermore, FLT3-TKD mutations in NK-AML did not impact the survival outcome [36]. Therefore, identification of new molecular abnormalities has revealed limitations associated with the prognostic information gained from cytogenetic profiling alone and has generated considerable interest in targeting these molecular lesions, such as FLT3-ITD, which contribute to poor outcomes.

\section{Existing treatment}

Although there has been rapid and significant progress in our understanding of the genetic basis of AML, limited progress has been seen in its treatment. Front-line therapy has changed very little over the last 3 decades. At the time of diagnosis, information from cytogenetic and molecular studies may not be available. Therefore, initial treatment decisions strongly depend on the patient's age and performance status. Current induction therapy for patients with AML (not including APL) and $<60$ years of age is comprised of idarubicin $\left(12 \mathrm{mg} / \mathrm{m}^{2}\right)$ or daunorubicin $\left(60-90 \mathrm{mg} / \mathrm{m}^{2}\right)$ intravenously over 3 days given concomitantly with 7 days of continuous infusion cytarabine $(7+3)$, followed by $2-4$ courses of remission consolidation with high-dose cytarabine or allogeneic stem cell transplant for eligible patients harboring poor-risk features. The $7+3$ regimen is capable of yielding complete response $(\mathrm{CR})$ rates of $\sim 60 \%$, but most patients ultimately relapse and die of their disease [37]. Recent data suggest that escalating the dose of daunorubicin to 80 or $90 \mathrm{mg} / \mathrm{m}^{2}$ can yield higher CR rates of 70 and $71 \%$, respectively [38,39]. However, the long-term impact of this dose intensification approach on survival is limited. To date, an anthracycline plus cytarabine remains first-line therapy for AML and modifications or the addition of agents to this combination have not yet yielded improved OS. For this reason, research endeavors continue to build on such regimens, most commonly by adding a third agent with non-overlapping toxicity.

Furthermore, AML occurs predominantly in elderly patients and this population has worse outcomes compared to younger patients. This may be due to poorer tolerance to therapy, higher frequency of poor cytogenetic features and multi-drug resistance expression [40]. Older patients experience a short median survival of $<1$ year $[41,42]$ and have $3-5$ year 
survival rates of $<10 \%$ [41,43-46]. Moreover, alternative low-intensity approaches, such as low-dose subcutaneous cytarabine, yield low CR rates (18\%) and a 30-day mortality rate of $26 \%$ [47]. A recent multivariate analysis of NK-AML patients older than 60 years treated on Cancer and Leukemia Group B (CALGB) frontline trials revealed a shorter DFS and OS in patients $60-69$ years of age harboring FLT3-ITD mutations [48]. The questionable benefits and tolerability of aggressive therapy in elderly patients and the lack of effective alternative treatment options have led to increased interest in exploring molecular-based therapies that would improve treatment outcomes and/or have a more favorable side effect profile.

Finally, patients who are refractory to front-line therapy or relapse within 1 year should be considered for a clinical trial and stem cell transplant [49]. Patients who experience relapse more than a year from the date of remission may receive standard front-line induction therapy and should be considered for stem cell transplant.

\section{Current research goals}

Recent advancements with the identification of new molecular defects and a better understanding of the physiological role these defects play in the evolution and survival of AML have improved the diagnostic and therapeutic potential for AML. Currently, cytogenetic and molecular profiling in AML is used to stratify patients into risk groups essential for treatment decisions. However, treatment strategies and clinical outcomes have largely remained unchanged over the last 3 decades. Successful targeting of adverse biological features with targeted agents has not been achieved for any subtype of AML, outside of APL. Therefore, continued emphasis should be placed on identifying key molecular abnormalities that adversely affect outcomes and the development of such agents that target them. Although advancements in the treatment of AML have been limited, current research goals should continue to focus on unraveling these complex biological features that contribute to the heterogeneous nature of the disease.

\section{Scientific rational}

The FLT3 receptor kinase is highly expressed in acute leukemias, and activating FLT3 mutations occur frequently in AML. Constitutive activation of FLT3 is caused by ITD mutations in the JM region (FLT3-ITD) or by point mutations in the second kinase domain (FLT3-TKD) [13,50,51]. A considerable amount of evidence suggests that FLT3-ITD mutations are associated with leukocytosis and increased peripheral and bone marrow blasts, and play an important role in the development and survival of leukemia cells. Although FLT3-ITD mutations may not impact rates of CR, substantial evidence suggests that FLT3ITD mutations confer a worse prognosis for patients with intermediate-risk cytogenetics (NK) $[30-32,36,48]$.

Point mutations also occur most frequently in patients with normal cytogenetics and are associated with proliferative disease, including increased bone marrow and peripheral blasts [30,31,52]. However, reports of the impact of point mutations on outcomes are conflicting; some suggesting poor outcomes, while others reporting favorable outcomes [30,31,53]. Similarly, wild-type FLT3 (FLT3-WT) can be constitutively activated through mechanisms involving overexpression of $F L T 3$ along with ligand stimulation via paracrine or autocrine 
signaling [54,55]. The impact FLT3-WT overexpression and the burden of mutated FLT3 on outcomes are unclear. Substantial interest in FLT3 as a therapeutic target has led to the development of several promising inhibitors of FLT3 that are in various stages of clinical development (Table 1) [56].

\section{Competitive environment}

\subsection{Sorafenib}

Sorafenib is a multikinase inhibitor approved for the treatment of metastatic renal cell carcinoma and unresectable hepatocellular carcinoma. In addition to solid tumors, sorafenib has been widely studied in the treatment of AML, due to its potent inhibition of FLT3 tyrosine kinase. Preclinical studies have shown sorafenib to inhibit multiple kinases [57,58] and induce dephosphorylation of Raf, MEK and ERK, and apoptosis in AML cells [59]. Additionally, sorafenib was 1000- to 3000-fold more effective at inducing growth arrest and apoptosis in $\mathrm{Ba} / \mathrm{F} 3$ cells with FLT3-ITD or FLT3-D835G mutations than in cells with D835Y or FLT3-WT. Furthermore, sorafenib reduced leukemia burden and prolonged survival in a mouse model [60].

Single-agent sorafenib for the treatment of relapsed or refractory AML was explored in several Phase I studies (Table 2). In general, sorafenib shows preferential selectivity for FLT3-ITD blasts compared to unmutated FLT3 [58,60-63]. Fifteen patients with refractory acute leukemia (12 AML, 2 ALL, 1 biphenotypic) were treated with single-agent sorafenib in a Phase I dose-escalation study [62]. The maximum tolerated dose (MTD) was determined at $400 \mathrm{mg}$ orally twice daily for 21 days of a 28 day cycle. The most common grade $\geq 3$ toxicities were neutropenic fever (33\%), fatigue (17\%) and hypokalemia (17\%). Although there were no complete or partial remissions observed, 11 (73\%) of 15 patients achieved stable disease as their best response. In all, $6(40 \%)$ of the 15 patients showed a reduction in bone marrow blasts, including the 2 patients with FLT3-ITD. Sorafenib produced sustained complete inhibition of FLT3 and ERK that was maintained throughout the treatment cycle [61]. In another Phase I study, 16 patients with relapsed or refractory AML were treated with sorafenib at different dose schedules [60]. Single-agent sorafenib significantly reduced the percentage of peripheral blood and bone marrow blasts in patients with FLT3-ITD. Of note, there were no responses in patients without this mutation. In addition to Phase I studies, several case reports with single-agent sorafenib have been reported and show promising results in patients with AML who harbor FLT3-ITD, including molecular remissions and complete resolution of leukemia cutis [64-67]. In particular, durable CRs and complete molecular remissions have been achieved with single-agent sorafenib in patients with relapsed AML after stem cell transplant [67].

Two studies have evaluated the feasibility, safety and efficacy of sorafenib in combination with standard induction and consolidation chemotherapy for newly diagnosed patients with AML (Table 3) $[68,69]$. Ravandi et al. recently published the results of a single-center Phase II study in which 51 patients, under the age of 60 years and irrespective of FLT3-mutation status, were treated with chemotherapy plus sorafenib [68]. Based on the results of the Phase I component of this trial in determining the MTD, sorafenib was dosed at $400 \mathrm{mg}$ orally twice daily on days $1-7$ during induction chemotherapy (idarubicin plus cytarabine) and 
then continuously with up to 5 cycles of consolidation chemotherapy (idarubicin plus cytarabine). Fifteen patients had FLT3 mutations (13 FLT3-ITD). The median age was 53 years. Overall, $75 \%$ of patients achieved a CR. Of the 15 FLT3-mutated patients, 14 (93\%) achieved CR (12/13 FLT3-ITD and 2/2 FLT3-TKD patients; the 13th FLT3-ITD patient had a CR with incomplete platelet recovery (CRp)). Significantly less CRs occurred in patients with FLT3-WT compared to FLT3-mutations (66 vs 93\%, respectively, $\mathrm{p}=0.033$ ). At a median of 54 weeks follow-up, the probability of survival at 1 year was $74 \%$. With a median follow-up of 62 weeks, 10 patients with FLT3-mutation relapsed and the other 5 patients remained in CR. The addition of sorafenib to chemotherapy was relatively well tolerated. Grade $\geq 3$ toxicities that were possibly related to sorafenib included hyperbilirubinemia (4 patients), elevated transaminases ( 5 patients), diarrhea (4 patients), rash ( 2 patients), pancreatitis (1 patient), colitis (1 patient), pericarditis (1 patient) and hand-foot syndrome (2 patients). Results of the plasma inhibitory assay (PIA) for 10 patients demonstrated complete inhibition of phosphorylated FLT3 by their day 7 plasma in FLT3-ITD, but not for FLT3-WT, cell lines [68].

The second trial conducted was a Phase II, multi-center, randomized, placebo-controlled, double-blind trial in elderly patients (> 60 years) with AML, irrespective of FLT3 status, treated with $7+3$ induction chemotherapy followed by consolidation with intermediate-dose cytarabine, with or without the addition of sorafenib (400 mg orally twice daily given between cycles) [69]. Patients randomized to sorafenib also received sorafenib as maintenance therapy. One-hundred and two patients received treatment with sorafenib and ninety-five patients received placebo. In all, 28 (14.2\%) of the 197 patients had FLT3-ITD mutations. CR or blast clearance without complete recovery of blood counts was seen in 48 and $8.8 \%$ of sorafenib patients and 60 and $4.2 \%$ of placebo patients, respectively. There was no significant difference in EFS or OS between the two groups (median EFS: 7 months placebo vs 5 months sorafenib, HR $1.261(\mathrm{p}=0.13)$; median OS: 15 months placebo vs 13 months sorafenib, HR $1.025(\mathrm{p}=0.89)$ ). Additionally, there was no difference in CR, EFS or OS in the small subgroup of FLT3-ITD patients. Sorafenib plus chemotherapy was relatively well tolerated. However, there was an increased incidence of infection-related deaths in the sorafenib arm of the study [69].

\subsection{Sunitinib (SU11248)}

Sunitinib is a multikinase [70], small molecule tyrosine kinase inhibitor (TKI) that was recently approved for the treatment of metastatic renal cell carcinoma and gastrointestinal stromal tumors not responsive to imatinib. Along with sorafenib, sunitinib is one of the most studied inhibitors of FLT3 in AML. In a Phase I study, O'Farrell et al. administered a single dose of sunitinib, escalated from 50 to $350 \mathrm{mg}$, to 29 patients with AML in cohorts of three to six [71]. Following administration of sunitinib, FLT3 phosphorylation and plasma pharmacokinetics (PK) were evaluated at multiple time points over $48 \mathrm{~h}$ and an analysis of FLT3 genotype was performed. Inhibition of FLT3 phosphorylation was observed in 50\% of FLT3-WT patients and in all of FLT3 mutated patients. Substantial inhibition of FLT3 phosphorylation in $>50 \%$ of the patients was observed with sunitinib doses of $200 \mathrm{mg}$ and higher. Drug-related adverse events occurred in $31 \%$ of the patients, but were limited to grade 1/2 diarrhea and nausea [71]. 
In another Phase I study, sunitinib was given to 15 relapsed or refractory AML patients at a starting dose of $50 \mathrm{mg} /$ day for 4 weeks followed by a 2- or 1-week rest period [72].

Although short in duration, partial responses (PRs) or better were seen in all 4 patients harboring FLT3 mutations as compared to 2 of 10 evaluable patients with FLT3-WT. Additionally, the mutated receptors were not detected at day 28 in two patients. The most frequent toxicities with $50 \mathrm{mg}$ a day consisted of grade 2 edema, fatigue and oral ulcerations. The $75 \mathrm{mg}$ dose level was abandoned after two patients each experienced grade 4 fatigue, hypertension and cardiac failure (Table 2). Exploiting the synergistic antileukemia effects demonstrated in vitro with sunitinib and chemotherapy on FLT3-ITD leukemia cells [73], Fiedler et al. explored, in a Phase I- II study, the safety and activity of sunitinib in combination with standard $7+3$ induction and consolidation therapy with intermediate doses of cytarabine $1 \mathrm{~g} / \mathrm{m}^{2}$ given on days 1,3 and 5 for 3 cycles [74]. Under the Phase I portion of the study, 12 patients evaluable for toxicity received either $50 \mathrm{mg}$ of sunitinib daily for 28 days or for the first 7 days during each cycle of induction and consolidation therapy. Eight patients harbored FLT3-ITD and four patients had FLT3-TKD mutations. The continuous dosing arm was abandoned due to dose-limiting toxicities in three of six patients that consisted of prolonged aplasia or hand- leg syndrome. The most common adverse events included grade 4 neutropenia and thrombocytopenia and infectious complications. Seven (70\%) of ten patients evaluable for response achieved a CR or a CR with incomplete recovery of the counts (CRi) and one patient experienced a PR. Three of four patients with FLT3-TKD mutation obtained a CR (Table 3).

\subsection{Lestaurtinib (CEP-701)}

Lestaurtinib, a small molecule FLT3 inhibitor, has been studied in several preclinical studies in both solid and hematologic malignancies. Lestaurtinib has demonstrated inhibition against the autophosphorylation of FLT3-ITD and -WT as well as cytotoxicity in leukemia cells, both in vivo and in vitro [75,76]. FLT3 inhibition with lestaurtinib resulted in improved survival in a mouse model [75]. Although the cytotoxicity of FLT3 inhibitors appears to be related to the inhibitory activity, there is great heterogeneity in responses. In general, it appears that blast cells with FLT3-ITD mutation are more sensitive to lestaurtinib compared to blast cells with a FLT3-TKD mutation or FLT3-WT, which is consistent with similar findings with other FLT3 inhibitors [76]. In addition, there is great variability in cytotoxic responses among patients with mutated or un-mutated FLT3 [76,77]. Furthermore, in vitro and in vivo studies showed synergistic cytotoxic effects in AML cell lines when lestaurtinib was given in combination with cytotoxic chemotherapy. Interestingly, this synergy was seen when lestaurtinib was administered simultaneously with or immediately following chemotherapy [76-78]. Conversely, studies have demonstrated antagonism with FLT3 inhibition when lestaurtinib was administered prior to chemotherapy [78].

The first Phase I - II study with lestaurtinib in AML treated 17 patients with relapsed or refractory AML and with activating mutations of FLT3 with a starting dose of $40 \mathrm{mg}$ orally twice daily [79]. The study was amended after the first three patients to increase the starting dose to $60 \mathrm{mg}$ twice daily with a planned escalation to $80 \mathrm{mg}$ twice daily due to suggested inadequate FLT3 inhibition by correlative studies. Five (36\%) of the fourteen patients had a response predominately with decreased peripheral blood blasts and decreased transfusion 
requirements. Although some clinical activity was demonstrated, responses were not durable, lasting from 2 to 12 weeks. However, correlative studies showed that all responders exhibited significant sustained FLT3 inhibition. Although this activity was seen at $60 \mathrm{mg}$ twice daily, the $80 \mathrm{mg}$ twice daily regimen may be associated with better sustained FLT3inhibitory plasma drug levels. In addition, lestaurtinib was safe and tolerable, with nonspecific and mild toxicities including nausea/vomiting, diarrhea, generalized weakness and fatigue (Table 2).

Knapper et al. conducted a Phase II trial of single-agent lestaurtinib for front-line treatment of older patients with AML not considered fit for intensive chemotherapy and irrespective of FLT3 mutation status [80]. Lestaurtinib was given orally for 8 weeks at a starting dose of 60 $\mathrm{mg}$ twice daily and escalated to $80 \mathrm{mg}$ twice daily on day 29 . Of the 27 evaluable patients, 8 (30\%) patients showed clinical activity. Overall, $3(60 \%)$ of the 5 patients with mutated FLT3 and 5 (23\%) of 22 patients with FLT3-WT demonstrated a response, which was predominately lowering of peripheral blasts and decreased transfusion dependence. Similar to the results of the Phase I study by Smith et al. [79], the majority of clinical responses were short lived with a median time to progression of 25 days ( $14-270$ days range). Correlative studies showed that clinical responses occurred when there was sustained FLT3inhibitory drug levels combined with in vitro cytotoxic sensitivity of blast cells to lestaurtinib. Overall, lestaurtinib was well tolerated and the most common reported toxicities included mild nausea/vomiting, constipation, diarrhea and an elevated alkaline phosphatase. The majority of patients tolerated dose escalation to $80 \mathrm{mg}$ twice daily (Table 3) [80].

In response to preclinical data showing synergistic activity with chemotherapy, Phase I- II data showing modest clinical activity with single-agent lestaurtinib, reported studies suggesting persistence of negative influence of FLT3-ITD on the outcome in relapse [81] and results of a Phase II trial showing clinical benefit of lestaurtinib in combination with chemotherapy in patients who were predicted to respond [82], Levis et al. initiated a Phase III, multi-center, randomized, open-label trial of salvage chemotherapy with or without lestaurtinib in patients with FLT3-mutated relapsed AML [83]. Chemotherapy consisted of mitoxantrone, etoposide and cytarabine or high-dose cytarabine depending on the duration of first remission. Lestaurtinib was dosed at $80 \mathrm{mg}$ orally twice daily and started 2 days after completion of chemotherapy. Although patients randomized to lestaurtinib were not tested for in vitro sensitivity prior to initiating chemotherapy, they had plasma samples obtained at baseline and days 15 and 42 to determine FLT3 inhibitory activity, and measurement of FL and a-1 acid glycoprotein (AAG). Two-hundred and twenty patients were treated and evaluated. The median age was 54 years in the chemotherapy alone arm and 59 years in the lestaurtinib arm; and the duration of CR1 was $<6$ months in $47 \%$ of patients in both arms. The majority of patients (88\%) had FLT3-ITD mutations. Lestaurtinib was generally well tolerated with comparable grade 3/4 adverse events in both arms (104 events in the lestaurtinib arm vs 101 in the chemotherapy alone arm, $p=0.8$ ). However, the incidence of serious infection and 30-day mortality were higher in the lestaurtinib arm compared to the chemotherapy alone arm (32 vs $21 \%$ and 12 vs $6 \%, \mathrm{p}=0.24$, respectively). In the intent-totreat analysis, there were $29(26 \%) \mathrm{CR} / \mathrm{CRp}$ in the lestaurtinib arm compared to $23(21 \%)$ in the control/chemotherapy alone arm $(\mathrm{p}=0.35)$. Additionally, there was no significant 
difference in OS between the two arms. Among the patients with target FLT3 inhibition (defined as PIA > 85\%) [84] at day $15(\mathrm{n}=46 / 79), 39 \%$ achieved CR/CRp compared to $9 \%$ of patients with less than target FLT3 inhibition. Interestingly, FL concentrations increased from baseline to day $15(15.6-1148 \mathrm{pg} / \mathrm{ml})$ and AAG concentrations rose by an average of $52 \%$ over the same time period. These PK factors may have reduced the degree of FLT3 inhibition and explain the negative results. The study was halted early due to failure to show improved clinical benefit when lestaurtinib was added to conventional chemotherapy (Table 3) [83].

\subsection{Midostaurin (PKC412)}

Midostaurin is a multitargeted TKI with demonstrated activity in AML and other hematologic and solid tumors. It was originally developed as a PKC inhibitor and underwent preclinical and Phase I studies in solid tumors. Midostaurin showed less than dose-limiting toxicity with $75 \mathrm{mg}$ orally three times daily in solid tumors [85]. It was found to have additional inhibitory activity against class III RTK. Weisberg et al. demonstrated midostaurin to induce cell toxicity in leukemia cells expressing mutant FLT3 and prolong OS in mice with FLT3-ITD leukemia [86]. Its inhibition of FLT3 made it an attractive agent for the treatment of patients with AML. Midostaurin produced concentration-dependent cytotoxic effects in FLT3-mutated cells and inhibited FLT3 phosphorylation in leukemia blasts [76]. Furthermore, in an effort to improve response rates and overcome resistance in FLT3-mutant AML cells, midostaurin has been studied in combination with several agents that target downstream signaling, such as the mTOR inhibitor rapamycin [87], the HDAC inhibitor LAQ824 [88] and the triterpenoid CDDO-Me [89], and found to work synergistically.

Stone et al. treated 20 patients with relapsed or refractory AML or high-risk MDS with documented FLT3 mutations with single-agent midostaurin at a dose of $75 \mathrm{mg}$ orally three times daily continuously [90]. The vast majority of patients had FLT3-ITD AML.

Midostaurin was generally well tolerated with mild nausea/vomiting as the most common toxicity. Although neutropenia was observed, especially in patients who responded, this may have also been related to disease itself. Of note, two patients experienced unexplained fatal pulmonary events, for which midostaurin could not be entirely excluded. Fourteen (70\%) of the twenty patients experienced peripheral blood blast reductions of $250 \%$, and $50 \%$ of these patients had a $\geq 2-\log$ reduction in peripheral blasts for at least 4 weeks. In all, 6 (30\%) of the 20 patients experienced bone marrow blast reductions by $\geq 50 \%$, including 1 patient who had a near CR. Unfortunately, responses were short in duration, lasting a median of 13 weeks (9 - 47 weeks). In PK studies, plasma concentrations of midostaurin increased substantially during the first week of treatment but then declined despite continuous dosing. This could be attributed to midostaurin's high affinity for protein binding, poor absorption or increased metabolism. This decline in concentrations of midostaurin and a metabolite (CGP2221) may be a contributing factor to the short duration of responses [90].

A subsequent Phase IIB study evaluated the safety and efficacy of single-agent midostaurin in patients with AML and high-risk MDS with either mutated- or wild type-FLT3 [91]. Ninety-five patients were randomized to receive midostaurin at either 50 or $100 \mathrm{mg}$ orally 
twice daily on a continuous basis. Overall, $89 \%$ of patients had AML and $73 \%$ of all patients were relapsed or refractory. Of the $37 \%$ patients with mutated FLT3, $74 \%$ had an ITD mutation. Thus, the majority of patients had unmutated FLT3 (FLT3-WT). Both patients with FLT3-mutation and WT responded to midostaurin. Of the 92 patients analyzed for efficacy, a blast response (defined as a reduction of $\geq 50 \%$ blasts in the bone marrow or peripheral blood) was achieved in 71\% of FLT3-mutated and 42\% of FLT3-WT patients. One patient with FLT3-mutation achieved a PR. Response rates were higher among untreated patients compared to relapsed or refractory patients, regardless of mutational status (100 vs $71 \%$, respectively, among FLT3-mutated; 50 vs 37\%, respectively, among FLT3-WT). Furthermore, the median time to treatment failure for patients who demonstrated a blast response, by Kaplan-Meier estimates, was 60 days in the FLT3-mutated group compared to 83 days in the FLT3-WT group. There was no difference in efficacy between the two doses. Midostaurin was generally well tolerated at both dose levels, with mild gastrointestinal-related toxicity being the most common. There were no fatal pulmonary events noted in this trial. This study confirmed the results of the proof-in-concept study by Stone et al. [90] and demonstrated modest activity with single-agent midostaurin (Table 2) [91].

In order to improve on response rates with single-agent midostaurin, a Phase Ib feasibility study of midostaurin (50 or $100 \mathrm{mg}$ ) in combination with induction and consolidation chemotherapy in patients < 61 years of age with newly diagnosed de novo AML was conducted by Stone et al. [92]. Patients could have either FLT-mutated or WT status. Patients received standard induction chemotherapy with the $7+3$ regimen followed by three cycles of consolidation with high-dose cytarabine, plus midostaurin twice daily on either days $8-21$ (sequential arm) or on days $1-7$ and $15-21$ (concomitant arm). The $50 \mathrm{mg}$ dose of midostaurin was selected as the dose for future studies due to poor patient tolerability of the $100 \mathrm{mg}$. Forty patients on the $50 \mathrm{mg}$ regimen were evaluated; twenty patients on each arm. A total of 27 patients had FLT3-WT and 13 were FLT3-mutated (9/13 had FLT3-ITD). Maintenance therapy with midostaurin was given to 5 patients (3 FLT3mutated). FLT3-mutated patients received a median of 133 days ( $21-975$ days) of midostaurin and FLT3-WT patients received a median of 90 days ( 7 - 1016 days). CRs occurred in $80 \%$ of all patients $(\mathrm{n}=40)$ and occurred more frequently in patients with FLT3mutation (92\%) compared to FLT3-WT (74\%). Unexpectedly, the 1 and 2 year OS rates for patients with FLT3-mutation ( 85 and 62\%, respectively) were similar to patients with FLT3WT (81 and 59\%, respectively) (Table 3) [92]. The results of this trial support the basis for the ongoing randomized, Phase III CALGB 10603 study in which newly diagnosed patients with AML, younger than 60 years of age with FLT3 mutations, receive induction/ consolidation with or without midostaurin and are further randomized to maintenance therapy with midostaurin or placebo.

\subsection{Tandutinib (MLN518)}

Tandutinib is another oral multi-target TKI selective for FLT3. In a Phase I trial evaluating the PK and pharmacodynamics (PD) of tandutinib, 40 patients with either AML or high-risk MDS were given tandutinib starting at $50 \mathrm{mg}$ orally twice a day, escalating in subsequent cohorts up to $700 \mathrm{mg}$ twice a day [93]. Two of five evaluable patients with FLT3-ITD who 
were treated with tandutinib 525 and $700 \mathrm{mg}$ twice daily demonstrated improvements in both blood and marrow blasts. The dose-limiting toxicities were reversible generalized muscle weakness, fatigue, or both and occurred at doses of 525 and $700 \mathrm{mg}$ twice a day in 1 of 6 and 2 of 3 patients, respectively [93]. DeAngelo et al. further studied tandutinib $525 \mathrm{mg}$ twice daily in a Phase II study in patients with relapsed or refractory AML with FLT3-ITD [94]. Of the 15 patients evaluable, 6 patients demonstrated $\geq 50 \%$ decreases in peripheral and bone marrow blasts of $1-3$ months duration (Table 2). In a Phase I- II trial, 29 newly diagnosed AML patients with or without FLT3 mutation were treated with tandutinib (200 $500 \mathrm{mg}$ twice daily) plus induction ( $3+7$ regimen) and consolidation (cytarabine) chemotherapy [95]. The most common drug-related adverse events were diarrhea, nausea and vomiting. The protocol was amended so that tandutinib was given on days $1-14$ of each cycle and this improved tandutinib tolerability. In all, 5 of the 7 patients treated with tandutinib $200 \mathrm{mg}$ twice daily continuously achieved a CR and 6/8 patients on tandutinib $200 \mathrm{mg}$ twice daily on days $1-14$ achieved CR (Table 3). In an ex vivo study, tandutinib displayed sequence-independent and strong synergistic anti-leukemic effects on FLT3-ITD blasts when combined with cytarabine and daunorubicin [96]. As suggested by the investigators, the strong synergistic effects seen on FLT3-ITD blasts may allow for exploring lower doses of chemotherapy in elderly patients [96].

\section{$6.6 \mathrm{AC220}$}

AC220 is a second generation, small molecule FLT3 inhibitor that displays an improved PK and PD profile over the first generation FLT3 inhibitors [97,98]. It has a favorable drug profile with low nanomolar potency, high tyrosine kinase selectivity and high and sustained FLT3 inhibition [97-102]. In animal studies, AC220 was able to achieve substantial and sustainable tyrosine kinase inhibition at achievable doses as low as $1 \mathrm{mg} / \mathrm{kg}$ orally daily $[97,100]$.

AC220 has been studied in several homozygous FLT3-ITD-dependent MV4-11 mouse xenograft models $[97-99,102,103]$. One study examined the efficacy of AC220 given as a limited treatment course (30 days) or a chronic dosing regimen [103]. The data suggest that AC220 is efficacious against both FLT3-ITD homozygous and heterozygous genotypes, and that a chronic dosing regimen may provide more efficacy compared to a limited treatment course. The effect of AC220 plus chemotherapy (cytarabine, cladribine, etoposide and daunorubicin) was examined against the homozygous FLT3-ITD cell line MV4-11 in an in vitro study [102]. When AC220 was given concurrently with or on the day following chemotherapy, it provided slightly synergistic effects. More importantly, unlike the results with lestaurtinib, AC220 given prior to the addition of chemotherapy did not result in significant antagonism. When AC220 was given, concurrently or episodically, with cytarabine or azacitidine to mice with solid tumors, it showed to be safe and have increased efficacy.

Cortes et al. conducted the first Phase I, dose-escalation study of AC220 in humans [100]. AC220 was administered orally at doses ranging from 12 to $450 \mathrm{mg}$ (flat dose) once daily for 14 days every 28 days to 76 relapsed or refractory AML patients with FLT3-ITD, FLT3WT or undetermined FLT3 mutations. Additional cohorts were studied on a continuous 
dosing regimen at 200 and $300 \mathrm{mg} /$ day for 28 days. PK and PD studies revealed AC220 plasma exposure to be sustained between dose intervals and to be dose-proportional. The half-life was 1.5 days, which allows for once-daily dosing. An active metabolite, AC886, was detected that has similar potency and activity to AC220. The $12 \mathrm{mg}$ dose potently inhibited phosphorylated FLT3 in ex vivo FLT3-ITD cell lines and complete inhibition of phosphorylated FLT3 in WT cell lines occurred at higher doses. AC220 suppressed phosphorylated FLT3, STAT5 and KIT in peripheral blasts. AC220 was generally well tolerated and the most common reported drug-related adverse events were $\leq$ grade 2 gastrointestinal toxicity, peripheral edema and dysgeusia. Two patients at $300 \mathrm{mg}$ (continuously) had grade 3 QTc prolongation but were also on concomitant medications known to prolong QT. Therefore, the DLT was observed at $300 \mathrm{mg}$ and the MTD was declared at $200 \mathrm{mg}$ continuously. Overall, 30\% (23/76) of patients had a response to AC220: $12 \%$ (9/76) CR including CRi and CRp; and 18\% (14/76) PR. The overall median duration of response and median survival was 14 weeks (4-62+ weeks; $1-68+$ weeks, respectively). Among the 18 patients with FLT3-ITD, 10 (56\%) responded. Additionally, on the $200 \mathrm{mg}$ continuous dose regimen, 4 of the 6 FLT3-ITD patients responded (1 CR, 1 CRp, 1 CRi and 1 PR). Response rates were lower among FLT3-WT patients (19\%) and FLT3- undetermined patients (36\%) (Table 2) [100].

The acceptable toxicity profile, favorable PK/PD properties and modest response rates of single-agent AC220 in heavily pretreated AML patients have led to ongoing Phase II studies of AC220 as monotherapy or in combination with chemotherapy in FLT3-ITD and FLT-WT AML patients.

\section{Potential development issues}

Several FLT3 inhibitors are being explored for the treatment of AML and have shown encouraging preclinical and clinical activity. However, at the same time, several limitations are emerging from the available experience. From a clinical perspective, the majority of patients who responded to treatment with single-agent FLT3 inhibitors only experienced transient improvements in the peripheral blood and bone marrow blasts. Possible reasons for poor efficacy may include: limitations in PK/PD characteristics such as insufficient sustained plasma concentrations, high protein binding, varying active metabolites, liver metabolism, and varying levels of potency and selectivity for FLT3 and emergence of resistant clones (Table 4 and Figure 1). The development of overt and progressive AML may require cooperation between at least two mutations. For example, activating mutations in FLT3 augment cellular proliferations and may cooperate with loss-of-function mutations such as PML/RARa $(\mathrm{t}(15 ; 17)$ or AML1/ETO $(\mathrm{t}(8 ; 21)$ that primarily impair hematopoietic differentiation and lead to subsequent apoptosis [1]. Based on this model, successful inhibition therapy may perhaps require a combination of agents that will lead to more durable control of the disease. Additionally, recent data suggest that prolonged exposure to FLT3 inhibitors will lead to resistant clones that use compensatory pathways for survival that are independent of FLT3 signaling [104]. Similar to point mutations in the BCR-ABL kinase domain in CML, mutations in the FLT3 kinase domain may play a role in acquired drug resistance. Furthermore, the hematopoietic microenvironment may add to resistance by rescuing leukemia cells and thereby making them less sensitive to FLT3 inhibitors [58]. 
Increased plasma FL levels after administration of chemotherapy have been reported and probably interfere with the clinical efficacy of FLT3 inhibitors when given after chemotherapy [105]. This may explain the modest clinical benefit seen when combination therapy was administered in Phase II and III trials, and will continue to be an area of intensive research. Hence, the transient and PRs observed with FLT3 inhibitors in AML, coupled with the discovery of drug-resistant leukemic blast cells, have made resistance to FLT3 inhibitors a growing concern. A better understanding behind the inherent and acquired mechanism of resistance will guide future drug development and generate novel approaches for overcoming resistance. Moreover, identification of patient populations (de novo vs refractory; FLT3-ITD vs -WT or -TKD; and high vs low mutant allelic burden) likely to respond differently to the various FLT3-inhibitors will remain an important area of research and development surrounding FLT3 inhibitor-based therapy.

\section{Conclusion}

FLT3 tyrosine kinase is highly expressed in leukemia cells, especially AML cells. More importantly, activating mutations of FLT3 are one of the most common molecular abnormalities in AML, and patients with NK-AML and FLT3-ITD mutations have a poor prognosis. Thus, inhibiting FLT3 and downstream pathways have become a large area of research in hopes of improving outcomes for this patient population. Several FLT3 inhibitors have been studied in preclinical and clinical trials. Although studies with singleagent therapy have mostly shown only modest activity, FLT3 inhibitors are under evaluation in combination therapy with standard chemotherapy, both in salvage and front-line settings.

\section{Expert opinion}

The identification of molecular abnormalities, such as FLT3-ITD, is providing further insight into the heterogeneity of AML and the response to treatment. For many decades, we predominately relied on cytogenetics for prognostic information and to aid with treatment decisions. For example, stem cell transplant in first CR is indicated for patients with complex (or unfavorable) cytogenetics, whereas it is not indicated for patients with favorable cytogenetics. However, we now know that patients with normal cytogenetics and FLT3-ITD have decreased DFS, remission duration and OS compared to patients without FLT3-ITD. Thus, risk-stratification in AML has shifted from a purely clinical-based system to a cytogenetic- and molecular-based system. Given the heterogeneity of AML and the modest response rates with FLT3 inhibitor therapy, sole inhibition of FLT3 is unlikely to have the same profound effect in AML as imatinib did in chronic myeloid leukemia. Clinical evidence with FLT3 inhibitors is showing us that successful inhibition of FLT3 will probably require not only novel agents, but novel combinations in order to achieve improved and durable clinical benefit. Great strides in understanding FLT3 and the development of FLT3 inhibitors have been made over the past decade. However, much remains to be discovered regarding the role of FLT3 inhibitors and who will best benefit from them. There are conflicting data surrounding the role of FLT3 inhibitor therapy for patients with and without activating mutations as well as in the front-line or relapsed setting. In general, patients with FLT3-ITD appear to have better responses to FLT3 inhibitors compared to those with FLT3-TKD or FLT3-WT. Similarly, all of the FLT3 inhibitors mentioned here 
are considerably less potent at inhibiting FLT3-WT (non-mutated) compared to FLT3-ITD, evidenced by their increased $\mathrm{IC}_{50}$ values $[97,106]$. Some studies suggest patients with $d e$ novo AML (first line) may have improved response rates with FLT3 inhibitors compared to relapsed or refractory patients [91], while others suggest the opposite [106]. The PK/PD profile of FLT3 inhibitors probably plays a significant role here, especially the degree of selectivity that the agent has. Additionally, allelic burden appears to play an important function in determining who may most likely benefit from FLT3 inhibitor-based therapy. Pratz et al. demonstrated that the FLT3-mutant allelic burden and the clinical status (de novo or relapsed) to be predictive of response [106]. Both relapsed AML samples and samples with a high mutant allelic burden were more likely to be responsive to cytotoxicity from FLT3 inhibition than those obtained from de novo or with low mutant allelic burden. The degree of FLT3 selectivity may play a role here as well. Thus, FLT3-inhibitors have a definite role in the treatment of patients with ITD mutations and potentially in patients with FLT3-WT.

Although the results of the studies combining FLT3 inhibitors with chemotherapy have been only modestly encouraging, successful inhibition of FLT3 mutant leukemic cells will most probably require combination therapy. Several ongoing randomized clinical trials are exploring the role of FLT3 inhibitors in various combined approaches. Whether FLT3 inhibitors will be best utilized in consolidation or as maintenance therapy will need further investigation.

With our growing knowledge about the impact that FLT3 has on prognosis on certain subgroups of patients, it certainly behooves us to continue pursuing novel agents that target these molecular abnormalities. Although several patients in clinical trials have demonstrated hematologic responses to various FLT3 inhibitors, few patients have experienced clinically meaningful and durable responses to date. The most impressive responses have been seen with sorafenib in the setting of relapse after stem cell transplant [67]. Similar to what we have seen in the treatment of CML with tyrosine kinases, prolonged exposure to FLT3 inhibitors will probably be necessary but may also select for resistant clones. If FLT3 inhibitors prove to be a worthy treatment option, they will most likely need to be combined with chemotherapy or other novel agents such as inhibitors that target signaling pathways downstream from FLT3 in order to prevent resistance.

\section{Acknowledgments}

F Ravandi has received research funding and honoraria from Bayer/Onyx and has received honoraria from Novartis. J Cortes has received research funding from Ambit Bioscience.

\section{Bibliography}

Papers of special note have been highlighted as either of interest $(\bullet)$ or of considerable interest $(\bullet)$ to readers.

1. Gilliland DG, Jordan CT, Felix CA. The molecular basis of leukemia. Hematology Am Soc Hematol Educ Program. 2004:80-97. [PubMed: 15561678]

2. Rosnet O, Birnbaum D. Hematopoietic receptors of class III receptor-type tyrosine kinases. Crit Rev Oncog. 1993; 4:595-613. [PubMed: 7506935] 
3. Rosnet O, Buhring HJ, Marchetto S, et al. Human FLT3/FLK2 receptor tyrosine kinase is expressed at the surface of normal and malignant hematopoietic cells. Leukemia. 1996; 10:238-48. [PubMed: 8637232]

4. McKenna HJ, Stocking KL, Miller RE, et al. Mice lacking flt3 ligand have deficient hematopoiesis affecting hematopoietic progenitor cells, dendritic cells, and natural killer cells. Blood. 2000; 95:3489-97. [PubMed: 10828034]

5. Mackarehtschian K, Hardin JD, Moore KA, et al. Targeted disruption of the flk2/flt3 gene leads to deficiencies in primitive hematopoietic progenitors. Immunity. 1995; 3:147-61. [PubMed: 7621074]

6. Maroc N, Rottapel R, Rosnet O, et al. Biochemical characterization and analysis of the transforming potential of the FLT3/FLK2 receptor tyrosine kinase. Oncogene. 1993; 8:909-18. [PubMed: 8384358]

7. Lyman SD, Jacobsen SE. c-kit ligand and Flt3 ligand: stem/progenitor cell factors with overlapping yet distinct activities. Blood. 1998; 91:1101-34. [PubMed: 9454740]

8. Tse KF, Mukherjee G, Small D. Constitutive activation of FLT3 stimulates multiple intracellular signal transducers and results in transformation. Leukemia. 2000; 14:1766-76. [PubMed: 11021752]

9. Rosnet O, Buhring HJ, deLapeyriere O, et al. Expression and signal transduction of the FLT3 tyrosine kinase receptor. Acta Haematol. 1996; 95:218-23. [PubMed: 8677746]

10. Zhang S, Fukuda S, Lee Y, et al. Essential role of signal transducer and activator of transcription (Stat)5a but not Stat5b for Flt3-dependent signaling. J Exp Med. 2000; 192:719-28. [PubMed: 10974037]

11. Mizuki M, Fenski R, Halfter H, et al. Flt3 mutations from patients with acute myeloid leukemia induce transformation of 32D cells mediated by the RAS and STAT5 pathways. Blood. 2000; 96:3907-14. [PubMed: 11090077]

12. Brandts C, Bulent S, Rode M. Constitutive activation of Akt by Flt3 internal tandem duplications is necessary for increased survival, proliferation, and myeloid transformation. Cancer Res. 2005; 65:9643-49. [PubMed: 16266983]

13. Nakao M, Yokota S, Iwai T, et al. Internal tandem duplication of the flt 3 gene found in acute myeloid leukemia. Leukemia. 1996; 10:1911-18. [PubMed: 8946930]

14. Drexler HG. Expression of FLT3 receptor and response to FLT3 ligand by leukemic cells. Leukemia. 1996; 10:588-99. [PubMed: 8618433]

15. Horiike S, Yokota S, Nakao M, et al. Tandem duplications of the FLT3 receptor gene are associated with leukemic transformation of myelodysplasia. Leukemia. 1997; 11:1442-6. [PubMed: 9305595]

16. Yokota S, Kiyoi H, Nakao M, et al. Internal tandem duplication of the FLT3 gene is preferentially seen in acute myeloid leukemia and myelodysplastic syndrome among various hematological malignancies. A study on a large series of patients and cell lines Leukemia. 1997; 11:1605-9.

17. Breitenbuecher F, Schnittger S, Grundler R, et al. Identification of a novel type of ITD mutations located in nonjuxtamembrane domains of the FLT3 tyrosine kinase receptor. Blood. 2009; 113:4074-7. [PubMed: 18483393]

18. Griffith J, Black J, Faerman C, et al. The structural basis for autoinhibition of FLT3 by the juxtamembrane domain. Mol Cell. 2004; 13:169-78. [PubMed: 14759363]

19. Scholl S, Fricke HJ, Sayer HG, Hoffken K. Clinical implications of molecular genetic aberrations in acute myeloid leukemia. J Cancer Res Clin Oncol. 2009; 135:491-505. [PubMed: 19125300]

20•. Gilliland DG, Griffin JD. The roles of FLT3 in hematopoiesis and leukemia. Blood. 2002; 100:1532-42. An excellent review on the roles of FLT3 in hematopoiesis and AML, and the rationale behind FLT3 as a therapeutic target in patients with AML. [PubMed: 12176867]

21. Yamamoto Y, Kiyoi H, Nakano Y, et al. Activating mutation of D835 within theactivation loop of FLT3 in human hematologic malignancies. Blood. 2001; 97:2434-9. [PubMed: 11290608]

22. Jiang J, Paez JG, Lee JC, et al. Identifying and characterizing a novel activating mutation of the FLT3 tyrosine kinase in AML. Blood. 2004; 104:1855-8. [PubMed: 15178581] 
23. Kindler T, Breitenbuecher F, Kasper S, et al. Identification of novel activation mutation (Y842C) within the activation loop of FLT3 in patients with acute myeloid leukemia (AML). Blood. 2005; 105:335-40. [PubMed: 15345593]

24. Frohling S, Scholl C, Levine RL, et al. Identification of driver and passenger mutations of FLT3 by high-throughput DNA sequence analysis and functional assessment of candidate alleles. Cancer Cell. 2007; 12:501-13. [PubMed: 18068628]

25. Reindl C, Bagrintseva K, Vempati S, et al. Point mutations in the juxtamembrane domain of FLT3 define a new class of activation mutations in AML. Blood. 2006; 107:3700-7. [PubMed: 16410449]

26. National Cancer Institute. [Accessed 01 September 2010] Surveillance epidemiology and end results stat facts sheets. cancer: acute myeloid leukemia. Available from: http://seer.cancer.gov/ statfacts/html/clyl.html

27. Estey E, Dohner H. Acute myeloid leukemia. Lancet. 2006; 368:1894-907. [PubMed: 17126723]

28. Byrd JC, Mrozek K, Dodge RK, et al. Pretreatment cytogenetic abnormalities are predictive of induction success, cumulative incidence of relapse, and overall survival in adult patients with de novo acute myeloid leukemia: results from Cancer and Leukemia Group B (CALGB 8461). Blood. 2002; 100:4325-36. [PubMed: 12393746]

29. Thiede C, Koch S, Creutzig E, et al. Prevalence and prognostic impact of NPM1 mutations in 1485 adult patients with acute myeloid leukemia (AML). Blood. 2006; 107:4011-20. [PubMed: 16455956]

30. Frohling S, Schlenk RF, Breitruck J, et al. Prognostic significance of activating FLT3 mutations in younger adults (16 to 60 years) with acute myeloid leukemia and normal cytogenetics: a study of the AML Study Group Ulm. Blood. 2002; 100:4372-80. [PubMed: 12393388]

31. Thiede C, Steudel C, Mohr B, et al. Analysis of FLT3-activating mutations in 979 patients with acute myelogenousleukemia: association with FAB subtypes and identification of subgroups with poor prognosis. Blood. 2002; 99:4326-35. [PubMed: 12036858]

32. Kottaridis PD, Gale RE, Frew ME, et al. The presence of a FLT3 internal tandemduplication in patients with acutemyeloid leukemia (AML) adds importantprognostic information to cytogeneticrisk group and response to the first cycleof chemotherapy: analysis of 854 patients from the United Kingdom Medical Research Council AML 10 and 12 trials. Blood. 2001; 98:1752-9. This evaluation confirmed the negative impact that FLT3-ITD mutations have on clinical outcomes in patients with AML. [PubMed: 11535508]

33. Oyarzo MP, Lin P, Glassman A, et al. Acute myeloid leukemia with $t(6 ; 9)$ (p23;q34) is associated with dysplasia and a high frequency of flt3 gene mutations. Am J Clin Pathol. 2004; 122:348-58. [PubMed: 15362364]

34. Gale RE, Hills R, Pizzey AR, et al. Relationship between FLT3 mutation status, biologic characteristics, and response to targeted therapy in acute promyelocytic leukemia. Blood. 2005; 106:3768-76. [PubMed: 16105978]

35. Schnittger S, Schoch C, Dugas M, et al. Analysis of FLT3 length mutations in 1003 patients with acute myeloid leukemia: correlation to cytogenetics, FAB subtype, and prognosis in the AMLCG study and usefulness as a marker for the detection of minimal residual disease. Blood. 2002; 100:59-66. This evaluation described patient characteristics common to have FLT-mutations, such as NK-AML, and showed FLT3 mutations to be associated with higher risk of relapse but not overall survival. [PubMed: 12070009]

36. Santos FP, Jones D, Qiao W, et al. Prognostic value of FLT3 mutationsamong different cytogenetic subgroups inacute myeloid leukemia. Cancer. 2010 Epub ahead of Print.

37. Lowenberg B, Downing JR, Burnett A. Acute myeloid leukemia. N Engl J Med. 1999; 341:105162. [PubMed: 10502596]

38. Fernandez HF, Sun Z, Yao X, et al. Anthracycline dose intensification in acute myeloid leukemia. N Engl J Med. 2009; 361:1249-59. [PubMed: 19776406]

39. Pautas C, Merabet F, Thomas X, et al. Randomized study of intensified anthracycline doses for induction and recombinant interleukin-2 for maintenance in patients with acute myeloid leukemia age 50 to 70 years: results of the ALFA-9801 study. J Clin Oncol. 2010; 28:808-14. [PubMed: 20048183] 
40. Appelbaum FR, Gundacker H, Head DR, et al. Age and acute myeloid leukemia. Blood. 2006; 107:3481-5. [PubMed: 16455952]

41. Estey E. Acute myeloid leukemia and myelodysplastic syndromes in older patients. J Clin Oncol. 2007; 25:1908-15. [PubMed: 17488990]

42. Rowe JM, Neuberg D, Friedenberg W, et al. Eastern Cooperative Oncology. A phase 3 study of three induction regimens and of priming with GM-CSF in older adults with acute myeloid leukemia: a trial by the Eastern Cooperative Oncology Group. Blood. 2004; 103:479-85. [PubMed: 14512295]

43. Kantarjian H, O'Brien S, Cortes J, et al. Results of intensive chemotherapy in 998 patients age 65 years or older with acute myeloid leukemia or high-risk myelodysplastic syndrome: predictive prognostic models for outcome. Cancer. 2006; 106:1090-8. [PubMed: 16435386]

44. Lang K, Earle C, Foster T, et al. Trends in the treatment of acute myeloid leukemia in the elderly. Drugs Aging. 2006; 22:943-55. [PubMed: 16323971]

45. de Lima M, Ghaddar H, Pierce S, Estey E. Treatment of newly-diagnosed acute myelogenous leukemia in patients aged 80 years and above. Br J Haematol. 1996; 93:89-93. [PubMed: 8611481]

46. Goldstone AH, Burnett A, Wheatley K, et al. Attempts to improve treatment outcomes in acute myeloid leukemia (AML) in older patients: the results of the United Kingdom Medical Research Council AML 11 trial. Blood. 2001; 98:1302-11. [PubMed: 11520775]

47. Burnett AK, Milligan D, Prentice AG, et al. A comparison of low-dose cytarabine and hydroxyurea with or without all-trans retinoic acid for acute myeloid leukemia and high-risk myelodysplastic syndrome in patients not considered fit for intensive treatment. Cancer. 2007; 109:1114-24. [PubMed: 17315155]

48. Whitman SP, Maharry K, Radmacher MD, et al. FLT3 internal tandem duplication associates with adverse outcome and gene- and microRNA-expression signatures in patients 60 years of age or older with primary cytogenetically normal acute myeloid leukemia: a Cancer and Leukemia Group B study. Blood. 2010; 116:3622-6. [PubMed: 20656931]

49. Ravandi F, Cortes J, Faderl S, et al. Characteristics and outcome of patients with acute myeloid leukemia refractory to 1 cycle of high-dose cytarabine-based induction chemotherapy. Blood. 2010; 116:5818-23. [PubMed: 20923968]

50. Yamamoto Y, Kiyoi H, Nakano Y, et al. Activating mutation of D835 within the activation loop of FLT3 in human hematologic malignancies. Blood. 2001; 97:2434-9. [PubMed: 11290608]

51. Abu-Duhier FM, Goodeve AC, Wilson GA, et al. Identification of novel FLT-3 Asp835 mutations in adult acute myeloid leukaemia. Br J Haematol. 2001; 113:983-8. [PubMed: 11442493]

52. Bacher U, Haferlach C, Kern W, et al. Prognostic relevance of FLT3-TKD mutations in AML: the combination matters-an analysis of 3082 patients. Blood. 2008; 111:2527-37. [PubMed: 17965322]

53. Yanada M, Matsuo K, Suzuki T, et al. Prognostic significance of FLT3 internal tandem duplication and tyrosine kinase domain mutations for acute myeloid leukemia: a meta-analysis. Leukemia. 2005; 19:1345-9. [PubMed: 15959528]

54. Hawley TS, Fong AZ, Griesser H, et al. Leukemic predisposition of mice transplanted with genemodified hematopoietic precursors expressing flt3 ligand. Blood. 1998; 92:2003-11. [PubMed: 9731058]

55. Drexler HG. Expression of FLT3 receptor and response to FLT3 ligand by leukemic cells. Leukemia. 1996; 10:588-99. [PubMed: 8618433]

56. Wiernik PH. FLT3 inhibitors for the treatment of acute myeloid leukemia. Clin Adv Hematol Oncol. 2010; 8:429-36. [PubMed: 20733555]

57. Wilhelm SM, Carter C, Tang L, et al. BAY 43-9006 exhibits broad spectrum oral antitumor activity and targets the RAF/MEK/ERK pathway and receptor tyrosine kinases involved in tumor progression and angiogenesis. Cancer Res. 2004; 64:7099-109. [PubMed: 15466206]

58. Mori S, Cortes J, Kantarjian H, et al. Potential role of sorafenib in the treatment of acute myeloid leukemia. Leuk Lymphoma. 2008; 49:2246-55. [PubMed: 19052971] 
59. Zhang W, Konopleva M, Ruvolo VR, et al. Sorafenib induces apoptosis of AML cells via Bimmediated activation of the intrinsic apoptotic pathway. Leukemia. 2008; 22:808-18. [PubMed: 18200035]

60•. Zhang W, Konopleva M, Shi YX, et al. Mutant FLT3: a direct target ofsorafenib in acute myelogenous leukemia. J Natl Cancer Inst. 2008; 100:184-98. This preclinical and clinical investigation of sorafenib in AML showed preferential activity against FLT3-ITD AML. [PubMed: 18230792]

61. Crump M, Hedley D, Kamel-ReidS S, et al. A randomized phase I clinical andbiologic study of two schedules ofsorafenib in patients with myelodysplasticsyndrome or acute myeloid leukemia: a NCIC (National Cancer Institute of Canada) Clinical Trials Group Study. Leuk Lymphoma. 2010; 51:252-60. [PubMed: 20109071]

62. Pratz KW, Cho E, Levis MJ, et al. A pharmacodynamic study of sorafenib in patients with relapsed and refractory acute leukemias. Leukemia. 2010; 24:1437-44. [PubMed: 20535150]

63. Borthakur G, Kantarjian H, Ravandi F, et al. Phase 1 study of sorafenib in patients with refractory or relapsed acute leukemias. Haematologica. 2010; 96:62-8. [PubMed: 20952518]

64. Safaian NN, Czibere A, Bruns I, et al. Sorafenib (Nexavar[registered]) induces molecular remission and regression of extramedullary disease in a patient with FLT3-ITD(+) acute myeloid leukemia. Leuk Res. 2009; 33:348-50. [PubMed: 18573526]

65. Lee SH, Paietta E, Racevskis J, et al. Complete resolution of leukemia cutis with sorafenib in an acute myeloid leukemia patient with FLT3-ITD mutation. Am J Hematol. 2009; 84:701-2. [PubMed: 19714594]

66•. Metzelder S, Wang Y, Wollmer E, et al. Compassionate use of sorafenib in FLT3-ITD-positive acute myeloid leukemia: sustained regression before and after allogeneic stem celltransplantation. Blood. 2009; 113:6567-71. A report of clinically meaningful responses induced by sorafenib when given pre- and post-stem cell transplant in patients with refractory, FLT3-ITD AML. [PubMed: 19389879]

67. Metzelder S, Finck A, Fey M, et al. Sorafenib monotherapy is effective in relapsed and refractory Flt3-ITD positive acute myeloid leukemia, particularly after allogeneic stem cell transplantation. Blood. 2010; 116 abstract 3314.

68. Ravandi F, Cortes JE, Jones D, et al. Phase I/II study of combination therapy with sorafenib, idarubicin, and cytarabine in younger patients with acute myeloidleukemia. J Clin Oncol. 2010; 28:1856-62. The first study that combined sorafenib with induction chemotherapy in the frontline setting. Combination therapy was safe and produced a high CR rate in FLT3-mutated patients. [PubMed: 20212254]

69. Serve H, Wagner R, Sauerland C, et al. Sorafenib in combination with standard induction and consolidation therapy in elderly AML patients: results from a randomized, placebo-controlled Phase II trial. Blood. 2010; 116 abstract 333.

70. Mendel DB, Laird AD, Xin X, et al. In vivo antitumor activity of SU11248, a novel tyrosine kinase inhibitor targeting VEGF and PDGF receptors: determination of a pharmacokinetic/ pharmacodynamic relationship. Clin Cancer Res. 2003; 9:327-37. [PubMed: 12538485]

71. O'Farrell AM, Foran JM, Fiedler W, et al. An innovative phase I clinical study demonstrates inhibition of FLT3 phosphorylation by SU11248 in acute myeloid leukemia patients. Clin Cancer Res. 2003; 9:5465-76. [PubMed: 14654525]

72. Fiedler W, Serve H, Dohner H, et al. A Phase 1 study of SU11248 in the treatment of patients with refractory or resistant acute myeloid leukemia (AML) or not amenable to conventional therapy for the disease. Blood. 2005; 105:986-93. [PubMed: 15459012]

73. Yee KW, Schittenhelm M, O'Farrell AM, et al. Synergistic effect of SU11248 with cytarabine or daunorubicin on FLT3 ITD-positive leukemia cells. Blood. 2004; 104:4202-9. [PubMed: 15304385]

74. Fiedler W, Krauter J, Gotze K, et al. A Phase I/II study combining sunitinib with standard ara-c/ daunorubicin chemotherapy in patients 60 years or older with FLT3 mutated AML. Blood. 2010; 116 abstract 3285.

75. Levis M, Allebach J, Tse KF, et al. A FLT3-targeted tyrosine kinase inhibitor is cytotoxic to leukemia cells in vitro and in vivo. Blood. 2002; 99:3885-91. [PubMed: 12010785] 
76. Knapper S, Mills KI, Gilkes AF, et al. The effects of lestaurtinib (CEP701) and PKC412 on primary AML blasts: the induction of cytotoxicity varies with dependence on FLT3 signaling in both FLT3-mutated and wild-type cases. Blood. 2006; 108:3494-503. [PubMed: 16868253]

77. Mead AJ, Gale RE, Kottaridis PD, et al. Acute myeloid leukemia blast cells with a tyrosine kinase domain mutation of FLT3 are less sensitive to lestaurtinib than those with FLT3 internal tandem duplication. Br J Haematol. 2008; 141:454-60. [PubMed: 18341639]

78••. Levis M, Pham R, Smith BD, Small D. In vitro studies of a FLT3 inhibitor combined with chemotherapy: sequence of administration is important to achieve synergistic cytotoxic effects. Blood. 2004; 104:1145-50. This in vitro study provided insight into the sequencing of chemotherapy and FLT3 inhibitors. Synergistic cytotoxicity occurred when lestaurtinib was given simultaneously or immediately after chemotherapy, whereas antagonism was observed when lestaurtinib was given prior to chemotherapy. [PubMed: 15126317]

79. Smith BD, Levis M, Beran M, et al. Single-agent CEP-701, a novel FLT3 inhibitor, shows biologic and clinical activity in patients with relapsed or refractory acute myeloid leukemia. Blood. 2004; 103:3669-76. [PubMed: 14726387]

80. Knapper S, Burnett AK, Littlewood T, et al. A phase 2 trial of the FLT3 inhibitor lestaurtinib (CEP701) as first-line treatment for older patients with acute myeloid leukemia not considered fit for intensive chemotherapy. Blood. 2006; 108:3262-70. [PubMed: 16857985]

81. Ravandi F, Kantarjian H, Faderl S, et al. Outcome of patients with FLT3-mutated acute myeloid leukemia in first relapse. Leuk Res. 2010; 34:752-6. [PubMed: 19878996]

82. Levis M, Smith BD, Beran M, et al. A randomized, open-label study of lestaurtinib (CEP-701), an oral FLT3 inhibitor, administered in sequence with chemotherapy in patients with relapsed AML harboring FLT3 activating mutations: clinical response correlates with successful FLT3 inhibition. Blood. 2005; 106 abstract 403.

83••. Levis M, Ravandi F, Wang ES, et al. Results from a randomized trial of salvage chemotherapy followed by lestaurtinib for patients with FLT3 mutant AML in first relapse. Blood. 2011 [Epub ahead of print] The first Phase III randomized trial with lestaurtinib in combination with chemotherapy in the salvage setting. This study was stopped early due to the lack of clinical benefit in patients receiving combination therapy.

84•. Levis M, Brown P, Smith BD, et al. Plasma inhibitory activity (PIA): a pharmacodynamic assay revealsinsights into the basis for cytotoxicresponse to FLT3 inhibitors. Blood. 2006; 108:347783. This study described PIA as a surrogate assay for monitoring the efficacy of FLT3 inhibitors. PIA is independent of PK properties such as protein binding and active metabolite concentrations. [PubMed: 16857987]

85. Propper DJ, McDonald AC, Man A, et al. Phase I and pharmacokinetic study of PKC412, an inhibitor of protein kinase C. J Clin Oncol. 2001; 19:1485-92. [PubMed: 11230495]

86. Weisberg E, Boulton C, Kelly LM, et al. Inhibition of mutant FLT3 receptors in leukemia cells by the small molecule tyrosine kinase inhibitor PKC412. Cancer Cell. 2002; 1:433-43. [PubMed: 12124173]

87. Mohi MG, Boulton C, Gu TL, et al. Combination of rapamycin and protein tyrosine kinase (PTK) inhibitors for the treatment of leukemias caused by oncogenic PTKs. Proc Natl Acad Sci USA. 2004; 101:3130-5. [PubMed: 14976243]

88. Bali P, George P, Cohen P, et al. Superior activity of the combination of histone deacetylase inhibitor LAQ824 and the FLT-3 kinase inhibitor PKC412 against human acute myelogenous leukemia cells with mutant FLT-3. Clin Cancer Res. 2004; 10:4991-7. [PubMed: 15297399]

89. Ahmad R, Liu S, Weisberg E, et al. Combining the FLT3 inhibitor PKC412 and the triterpenoid CDDO-Me synergistically induces apoptosis in acute myeloid leukemia with the internal tandem duplication mutation. Mol Cancer Res. 2010; 8:986-93. [PubMed: 20571062]

90•. Stone RM, DeAngelo DJ, Klimek V, et al. Patients with acute myeloidleukemia and an activating mutation in FLT3 respond to a small-molecule FLT3 tyrosine kinase inhibitor, PKC412. Blood. 2005; 105:54-60. This Phase II proof-of-concept trial showed single-agent midostaurin to produce short-lived clinical responses in patients with mutated FLT3 refractory AML. This trial led to combination studies with chemotherapy. [PubMed: 15345597]

91 . Fischer T, Stone RM, DeAngelo DJ, et al. Phase IIB trial of oral Midostaurin (PKC412), the FMS-like tyrosine kinase 3 receptor (FLT3) and multi-targeted kinase inhibitor, inpatients with 
acute myeloid leukemia andhigh-risk myelodysplastic syndrome witheither wild-type or mutated FLT3. J Clin Oncol. 2010; 28:4339-45. This Phase IIb trial confirmed the results of the proof-inconcept study and gave support to investigations of midostaurin in combination with chemotherapy. [PubMed: 20733134]

92. Stone RM, Fischer T, Paquette R, et al. Phase 1b study of midostaurin (PKC412) in combination with daunorubicin and cytarabine induction and high-dose cytarabine consolidation in patients under age 61 with newly diagnosed de novo acute myeloid leukemia: overall survival of patients whose blasts have FLT 3 mutations is similar to those with wild-type FLT3. Blood. 2009; 114 abstract 634 .

93•. DeAngelo DJ, Stone RM, Heaney ML, et al. Phase 1 clinical results with tandutinib (MLN518), a novel FLT3 antagonist, in patients with acute myelogenous leukemia or high-risk myelodysplastic syndrome: safety, pharmacokinetics, and pharmacodynamics. Blood. 2006; 108:3674-81. This Phase I trial showed tandutinib to be well tolerated and generate modest clinical activity in refractory AML patients. [PubMed: 16902153]

94. DeAngelo DJ, Stone RM, Heaney ML, et al. Phase II evaluation of the tyrosine kinase inhibitor MLN518 in patients with acute myeloid leukemia (AML) bearing a FLT3 internal tandem duplication (ITD) mutation. Blood. 2004; 104 abstract 1792.

95. DeAngelo DJ, Amrein PC, Kovacsovics TJ, et al. Phase 1/2 study of tandutinib (MLN518) plus standard induction chemotherapy in newly diagnosed acute myelogenous leukemia (AML). Blood. 2006; 108 abstract 158.

96. Schittenhelm MM, Kampa KM, Yee KW, et al. The FLT3 inhibitor tandutinib (formerly MLN518) has sequence-independent synergistic effects with cytarabine and daunorubicin. Cell Cycle. 2009; 8:2621-30. [PubMed: 19625780]

97. Zarrinkar PP, Gunawardane RN, Cramer MD, et al. AC220 is a uniquely potent and selective inhibitor of FLT3 for the treatment of acute myeloid leukemia (AML). Blood. 2009; 114:2984-92. [PubMed: 19654408]

98. Levis M, Sato T, Murphy K, et al. FLT3 mutant to wild type allelic ratio and clinical status are predictive of response to FLT3 inhibitors in AML. Blood. 2009; 114 abstract 1716.

99. Chao Q, Sprankle KG, Grotzfeld RM, et al. Identification of N-(5-tert-butyl-isoxazol-3-yl)-N'-\{4[7-(2-morpholin-4-yl-ethoxy)imidazo[2,1-b] [1,3] benzothiazol-2-yl]phenyl \}urea dihydrochloride (AC220), a uniquely potent, selective, and efficacious FMS-like tyrosine kinase-3 (FLT3) inhibitor. J Med Chem. 2009; 52:7808-16. [PubMed: 19754199]

100• Cortes J, Foran J, Ghirdaladze D, et al. AC220, a potent, selective, second generation FLT3 receptor tyrosine kinase (RTK) inhibitor, in a first-in-human (FIH) phase 1 AML study. Blood. 2009; 114 abstract 636. This Phase I study described the PK characteristics of AC220, determined the MTD for future studies, and demonstrated ACC 220 to produce response rates in refractory AML patients.

101. James J, Pratz K, Stine A, et al. Clinical pharmacokinetics and FLT3 phosphorylation of AC220, a highly potent and selective inhibitor of FLT3. Blood. 2008; 112 abstract 2637.

102. Belli BA, Dao A, Bhagwat S, et al. AC220, a potent and specific FLT3 inhibitor, enhances the cytotoxic effects of chemotherapeutic agents in cell culture and in mouse tumor xenografts. Blood. 2009; 114 abstract 2052.

103. Brigham D, Belli BA, Breider M, et al. AC220, a FLT3 inhibitor, increases survival in two genotypically distinct FLT3-ITD models of acute myeloid leukemia and provides sustained protection following chronic administration. Blood. 2009; 114 abstract 2053.

104. Piloto O, Wright M, Brown P, et al. Prolonged exposure to FLT3 inhibitors leads to resistance via activation of parallel signaling pathways. Blood. 2007; 109:1643-52. [PubMed: 17047150]

105. Sato T, Yang X, Knapper S, et al. FLT3 ligand impedes the efficacy of FLT3 inhibitors in vitro and in vivo. Blood. 2011 Epub ahead of print.

106••. Pratz KW, Sato T, Murphy KM, et al. FLT3-mutant allelic burden and clinical status are predictive of response to FLT3 inhibitors in AML. Blood. 2010; 115:1425-32. This study highlights the significance of FLT3-mutant allelic burden and clinical status on response to FLT3 inhibitors; high mutant allelic burden and patients with relapsed disease were associated with higher response to cytotoxicity with FLT3 inhibitors. It also provides important information on 
the relationship between FLT3 inhibitor selectivity and response to treatment. [PubMed: 20007803] 

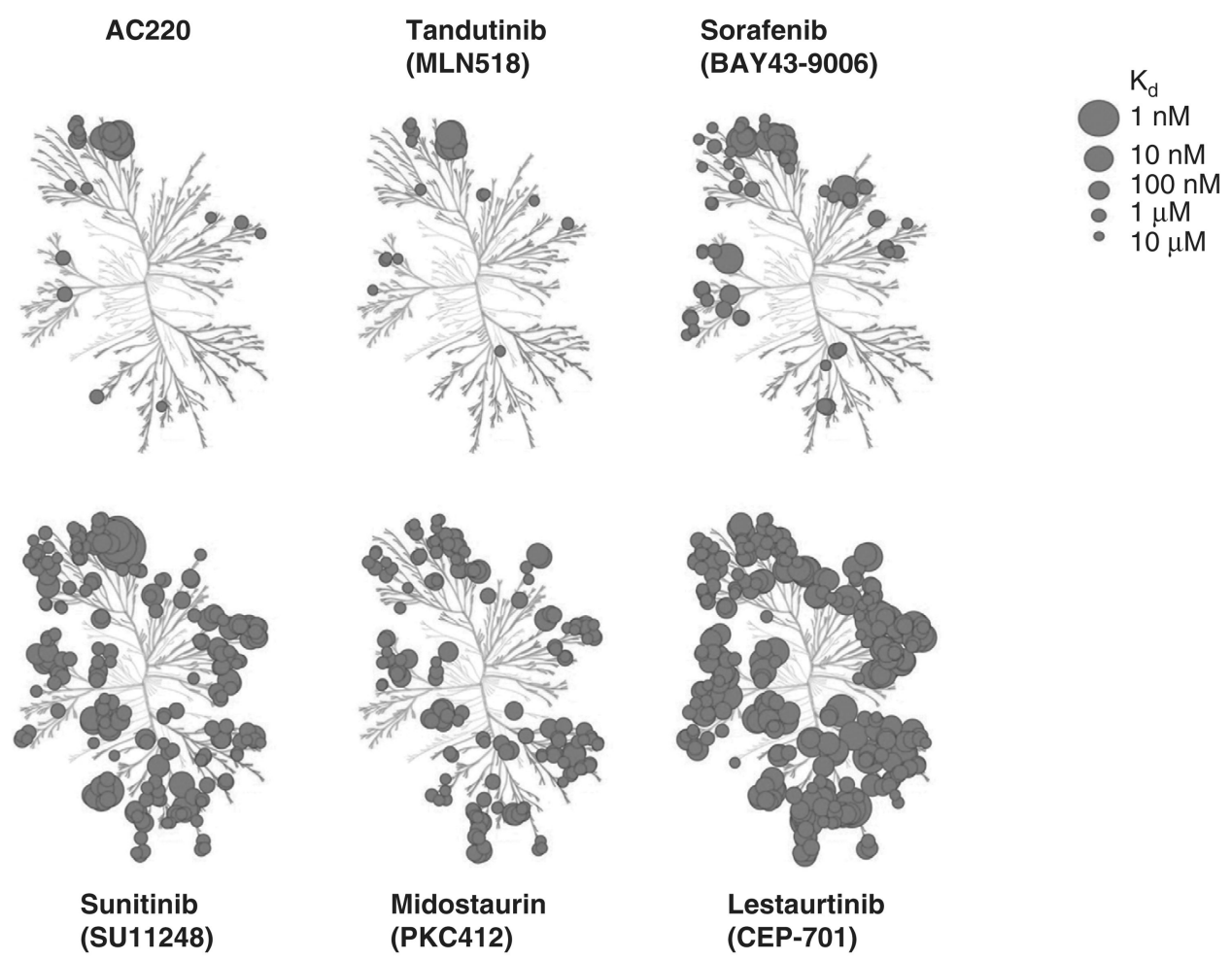

Figure 1. Kinase selectivity of FLT3 inhibitors

This kinase dendogram was adapted and is reproduced with permission from the American Society of Hematology [97].

FLT3: FMS-like tyrosine kinase 3. 


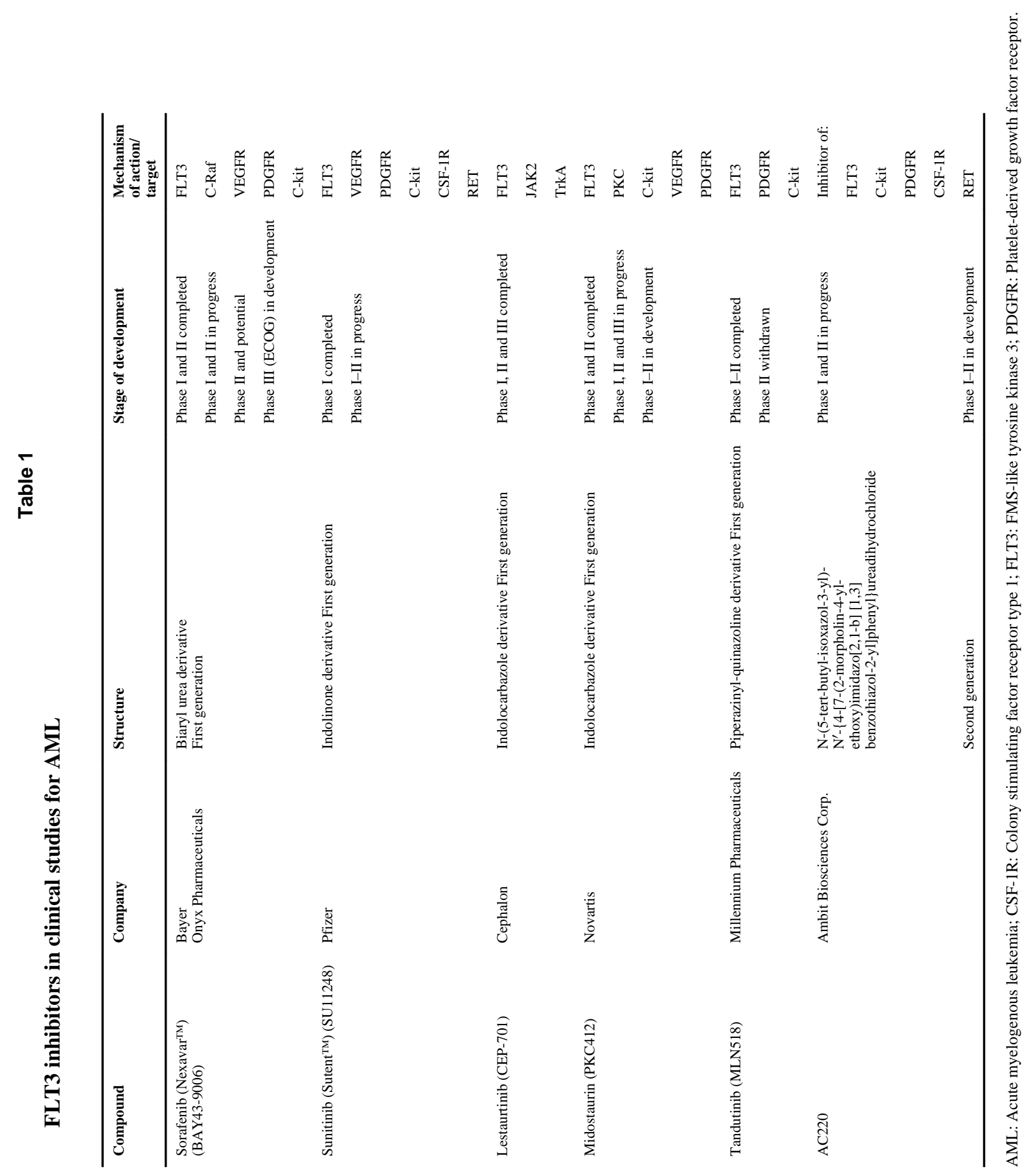




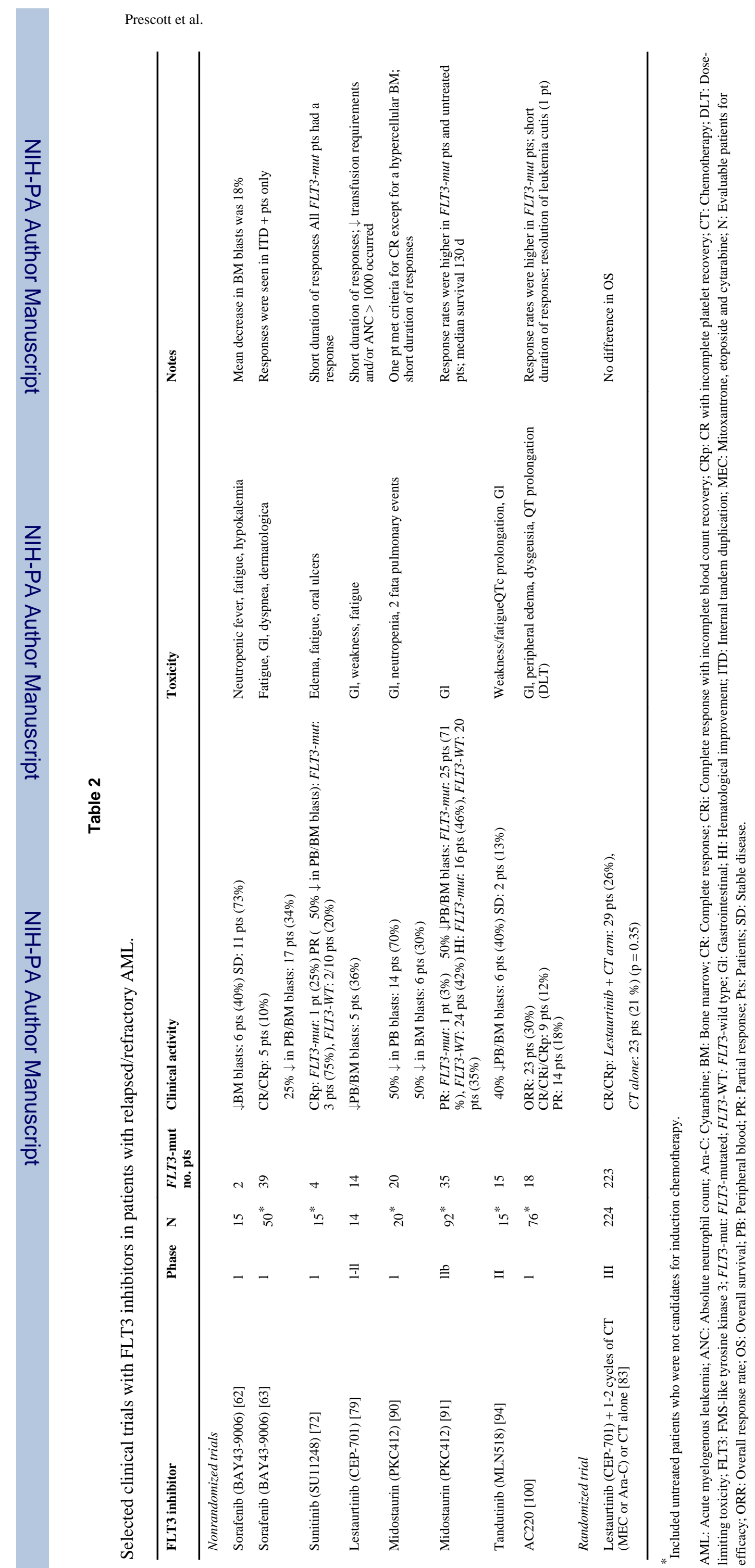




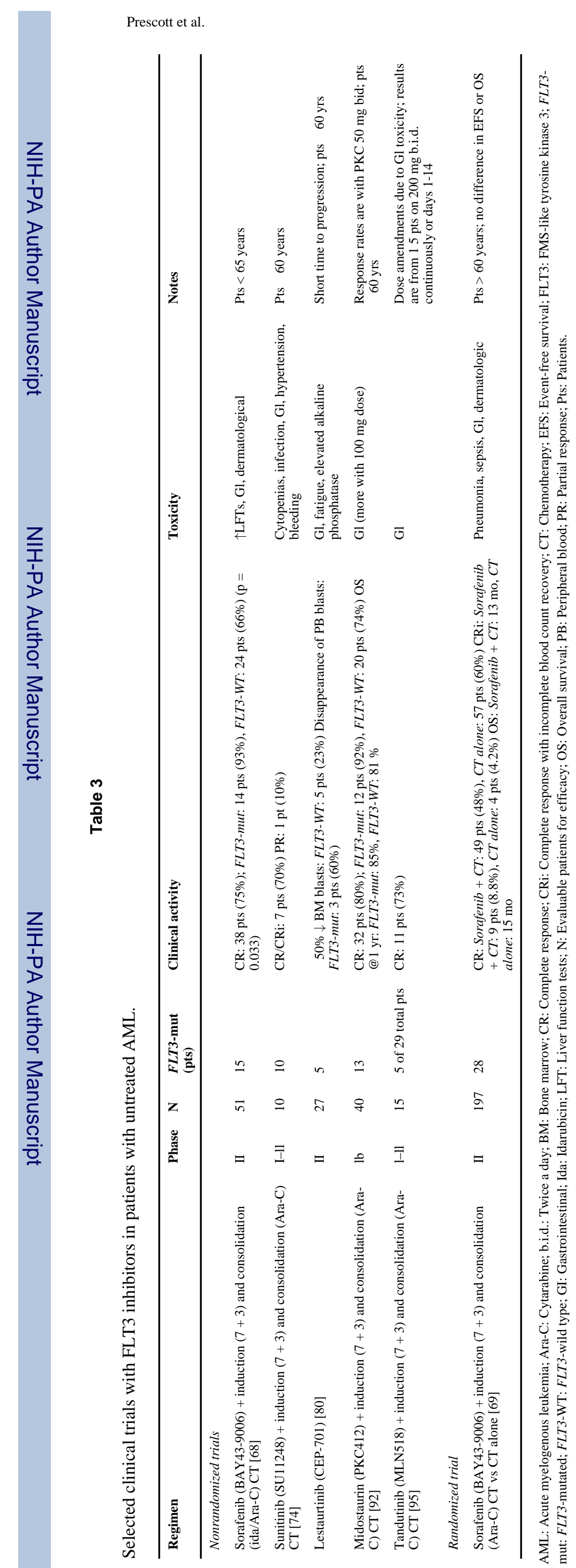

Page 25 
Table 4

Potency of FLT3 inhibitors

\begin{tabular}{|c|c|c|c|}
\hline FLT3-inhibitor & $\begin{array}{l}\text { FLT3-ITD autophorphorylation, } \mathrm{IC}_{50} \text {, } \\
\text { medium }(\mathrm{nM})\end{array}$ & $\begin{array}{l}\text { FLT3-ITD autophorphorylation, } \mathrm{IC}_{50} \text {, } \\
\text { plasma (nM) }\end{array}$ & Ref. \\
\hline AC220 & 1 & 18.4 & {$[97,98,106]$} \\
\hline Tandutinib (MLN518) & 6 & $\sim 200$ & {$[93,96]$} \\
\hline Sorafenib (BAY43-9006) & 3 & $308-484$ & {$[62,106]$} \\
\hline Sunitinib (SU11248) & 1 & NR & {$[98,106]$} \\
\hline Midostaurin (PKC412) & $3-6$ & $1000-1800$ & {$[84,106]$} \\
\hline Lestaurtinib (CEP-701) & 2 & 700 & {$[98,106]$} \\
\hline
\end{tabular}

FLT3: FMS-like tyrosine kinase 3; ITD: Internal tandem duplication; NR: Not reported. 